\title{
Why does bone have TERM limits?
}

\author{
C. H. Evans ${ }^{1}$ and M. J. Stoddart ${ }^{2,3}$
}

${ }^{1}$ Rehabilitation Medicine Research Center, Mayo Clinic, Rochester, MN, USA

${ }^{2}$ AO Research Institute Davos, Davos Platz, Switzerland

${ }^{3}$ Medical Faculty, Albert Ludwigs University of Freiburg, Freiburg, Germany

Keywords: osteogenesis; bone healing; tissue engineering; regenerative medicine; bone morphogenetic protein; growth factor; biomechanics; animal model

Address for correspondence:

C. H. Evans, PhD

Rehabilitation Medicine Research Center, Mayo Clinic, 200, First Street SW, Rochester MN, USA

Tel: + 15072550099

Evans.christopher@mayo.edu 


\section{Why does bone have TERM limits?}

\section{Introduction}

Bone, we are frequently reminded, is one of the few human organs with the ability to heal scarlessly and restore full function after injury. On this basis it should provide low hanging fruit for tissue engineering and regenerative medicine (TERM). But it doesn't. Instead, bone is proving a surprisingly stubborn target for TERM such that autografting, introduced in the 1950s, remains the clinical modality of choice. There is much discussion among researchers of the need for better scaffolds, delivery devices and sources of progenitor cells as agents of improved bone induction ${ }^{1}$. Here, we contribute to the conversation in a more general way, by examining strategic constraints to progress. The emphasis given below on BMP-2 reflects its widespread clinical use.

\section{Choice of pre-clinical species}

Understandably, most experimental work is performed with rodents. Unlike humans, however, rats and mice respond extremely well to recombinant, human bone morphogenetic protein (BMP-2) ${ }^{2}$. So do rabbits ${ }^{3}$ and dogs ${ }^{4}$. This discrepancy is reflected at the cellular level. Cultures of mesenchymal stromal cells (MSCs) grown from the bone marrow of rats and mice differentiate into osteoblasts and deposit mineral in response to BMP- $2^{5}$. Those grown from human bone marrow respond to BMP-2 by expressing only some of the genes associated with osteogenesis and do not deposit mineral ${ }^{6}$. Moreover, although the inflammatory cytokines interleukin-1 and tumour necrosis factor- $\alpha$ inhibit the osteogenic differentiation of rodent MSCs

7 they enhance mineralisation and other aspects of the osteogenic differentiation of human MSCs ${ }^{8}$. Ripamonte ${ }^{9}$ argues forcefully for the use of baboons in pre-clinical studies.

Such matters are not unique to bone healing. In a 2013 PNAS paper, Seok et al ${ }^{10}$ addressed the use of murine models of human inflammation to develop potential drugs, and commented:

"To date, there have been nearly 150 clinical trials testing candidate agents intended to block the inflammatory response in critically ill patients, and every one of these trials failed" 


\section{Maturity of Laboratory Animals}

Compounding concerns about species is the questionable skeletal maturity of the rodents used in research, whose growth plates never close. Some investigators instead use slowing of longitudinal bone growth as a criterion of skeletal maturity. Horton et al ${ }^{11}$, for instance, noted that the growth rate of the Sprague-Dawley tibia began to decline at day 64 and was essentially zero by day 109 in females and day 144 in males. After evaluating a variety of criteria, Sengupta

12 concluded that one rat month is comparable to three human years. Whatever the reality, it is clear that young, inbred, healthy rodents, who do not smoke or drink, are used for most laboratory studies and may not reflect the target human population.

\section{Scale-up is a problem}

Regardless of the intrinsic biological differences between small laboratory animals and humans, the issue of scaling is particularly problematic. Much of this reflects the high oxygen demand of bone and the absolute need for angiogenesis. To some degree the strategy of promoting the endochondral route to bone formation obviates this concern, especially as chondrogenesis is favoured by hypoxia. However, it remains the case that most cells need to be within $1 \mathrm{~mm}$ of a blood vessel to receive adequate oxygen. This is a challenge for a large segmental defect of human proportions, whose volume can exceed $30 \mathrm{cc}$. Large animals can also bring surgical problems; sheep, for instance, are notorious for breaking the hardware used to stabilize large osseous defects. This is challenging because the mechanical environment is a major driver in bone healing, dramatically influencing which healing pathway is initially taken and how it responds to growth factors. Moreover mundane constraints, such as cost and availability, cannot be overlooked.

\section{Not all bones are equal}

The opening sentence of this editorial refers to the long bones. Cranial defects, on the contrary, do not heal in individuals over the age of 2 years. Unlike the long bones, cranial bones do not bear load and do not depend upon loading to maintain mineral density and structural integrity. They form intra-membraneously, not endochondrally, during development. Cranial defects are 
widely used as convenient models of bone healing that require no fixation. While appropriate for developing better ways to heal cranial defects in people, they may have limited applicability to human long bone healing. Likewise, the healing of segmental defects in long bones is unlikely to follow the same initial biology as fracture healing. The formation of bone in a heterotopic location, widely used in experimental studies, is a promising initial screen but little more. In vitro testing is helpful, but the mechanism of bone formation in vivo is often overlooked in such studies. Potentially osteogenic materials are frequently tested using osteogenic media, which are relevant to intra-membraneous ossification but not endochondral ossification, the more common route in vivo.

\section{Excessive focus on BMP-2}

Most laboratory studies attempt to improve the performance of recombinant, human BMP-2 as the agent of bone healing. This is understandable, because it is one of only two BMPs approved by the FDA and EMA for clinical use and is readily available. However, in some ways it is reminiscent of the old story where someone who lost their car keys in the distant, dark woods looks for them under a nearby lamp-post because it's easier to see there. Recombinant BMP-2 is

a dimeric protein that does not exist in nature ${ }^{13}$. The native form is a heterodimer comprising one chain of BMP-2 and one of another BMP, such as BMP-7. Unlike recombinant BMP-2, the BMP-2/7 heterodimer is resistant to noggin, and other inhibitors of BMP-2 that impair its activities. BMP-6 is also resistant to these inhibitors ${ }^{14}$ and might bear increased scrutiny as an alternative to BMP-2. Moreover, there are a number of additional promising, but largely ignored, growth factors that individually or collectively contribute to osteogenesis. Ripamonti ${ }^{9}$, for example, has emphasised the importance of transforming growth factor $\beta_{3}$ in this regard. A number of osteogenic peptides and small molecules provide alternative opportunities.

\section{More is not necessarily better}

A popular strategy to improve bone healing involves delivering as much growth factor as possible to the defect site. Indeed, had not safety concerns intervened, the product AMPLIFY, using $2 \mathrm{mg} / \mathrm{cc}$ of recombinant, human BMP-2 rather than the $1.5 \mathrm{mg} / \mathrm{cc}$ of INFUSE, would have been launched for spinal fusions. But high doses of BMP-2 are inflammatory, trigger osteoclastic 
activity, engender heterotopic ossification and raise concerns about malignancy ${ }^{15}$. Moreover because of receptor dimerization, excessive concentrations of growth factors can lead to a saturation effect, reducing intracellular signaling. It is often commented that the doses of recombinant BMP-2 used clinically and in animal models are orders of magnitude greater than those occurring physiologically. This is likely to be true for other factors if not delivered at the correct dose at the correct time.

\section{Basic knowledge is lacking}

Perhaps the most fundamental problem is our lack of deep knowledge about the basic biology of the problems we are trying to solve. Although the developmental biology of bone and the biology of fracture healing are quite well understood, at least in certain laboratory animals, we know little of why non-unions develop and why segmental defects beyond a certain size will not heal spontaneously in young healthy individuals who would otherwise heal a fracture unproblematically.

When delivering an osteogenic agent we do not know how much is needed or when. Certain data

${ }^{16}$, for instance, suggest that delayed delivery could be an advantage and, as noted above, too much BMP-2 can be as bad as too little. Without dosing and timing information, it is not possible to develop the commensurate delivery strategies. Moreover, there are likely to be complex interactions between the biology and mechanics of healing that will influence outcome. These interactions have not been studied in detail.

\section{Conclusions}

It is often easier to identify problems than to solve them, and such is the case here. The issue of which pre-clinical species to use is particularly troublesome, because animals larger than rats and mice are expensive and often of limited availability. However, only mice provide opportunities for studies requiring sophisticated genetic manipulation or a large selection of molecular and immunological probes. More predictive in vitro screens would help considerably in this regard. Scale up remains a particularly difficult issue, especially as several candidate species are companion animals and the use of non-human primates is increasingly restricted. Their need 
could be reduced if the authorities would permit early, human, clinical trials, once safety has been established. Greater use of veterinary patients in clinical trials would also help ${ }^{17}$.

Most of the other issues raised here seem addressable by increased attention to basic, mechanistic research. Here the rate-limiting step is funding, discussion of which lies beyond the scope of this editorial.

Finally, as much as BMP-2 is maligned, it does add to the trauma surgeon's armamentarium, even if incrementally. And there are not a lot of other options. As commented by Cheng et al: "If not BMP, then what?" 18.

\section{References}

1. Verrier S, Alini M, Alsberg E, et al. 2015. Tissue Engineering and Regenerative Approaches to Improving the Healing of Large bone Defects. European Cells and Materials In Press.

2. Liu F, Wells JW, Porter RM, et al. 2016. Interaction between living bone particles and rhBMP-2 in large segmental defect healing in the rat femur. J Orthop Res.

3. Zegzula HD, Buck DC, Brekke J, et al. 1997. Bone formation with use of rhBMP-2 (recombinant human bone morphogenetic protein-2). J Bone Joint Surg Am 79:1778-1790. 4. Boyce AS, Reveal G, Scheid DK, et al. 2009. Canine investigation of rhBMP-2, autogenous bone graft, and rhBMP-2 with autogenous bone graft for the healing of a large segmental tibial defect. J Orthop Trauma 23:685-692.

5. Balk ML, Bray J, Day C, et al. 1997. Effect of rhBMP-2 on the osteogenic potential of bone marrow stromal cells from an osteogenesis imperfecta mouse (oim). Bone 21:7-15.

6. Diefenderfer DL, Osyczka AM, Garino JP, Leboy PS. 2003. Regulation of BMP-induced transcription in cultured human bone marrow stromal cells. J Bone Joint Surg Am 85-A Suppl 3:19-28.

7. Lacey DC, Simmons PJ, Graves SE, Hamilton JA. 2009. Proinflammatory cytokines inhibit osteogenic differentiation from stem cells: implications for bone repair during inflammation. Osteoarthritis Cartilage 17:735-742.

8. Ferreira E, Porter RM, Wehling N, et al. 2013. Inflammatory cytokines induce a unique mineralizing phenotype in mesenchymal stem cells derived from human bone marrow. J Biol Chem 288:29494-29505.

9. Ripamonti U, Duarte R, Ferretti C. 2014. Re-evaluating the induction of bone formation in primates. Biomaterials 35:9407-9422.

10. Seok J, Warren HS, Cuenca AG, et al. 2013. Genomic responses in mouse models poorly mimic human inflammatory diseases. Proc Natl Acad Sci U S A 110:3507-3512. 
11. Horton JA, Bariteau JT, Loomis RM, et al. 2008. Ontogeny of skeletal maturation in the juvenile rat. Anat Rec (Hoboken) 291:283-292.

12. Sengupta P. 2013. The Laboratory Rat: Relating Its Age With Human's. Int J Prev Med 4:624-630.

13. Israel DI, Nove J, Kerns KM, et al. 1996. Heterodimeric bone morphogenetic proteins show enhanced activity in vitro and in vivo. Growth Factors 13:291-300.

14. Song K, Krause C, Shi S, et al. 2010. Identification of a key residue mediating bone morphogenetic protein (BMP)-6 resistance to noggin inhibition allows for engineered BMPs with superior agonist activity. J Biol Chem 285:12169-12180.

15. Carragee EJ, Hurwitz EL, Weiner BK. 2011. A critical review of recombinant human bone morphogenetic protein-2 trials in spinal surgery: emerging safety concerns and lessons learned. Spine J 11:471-491.

16. Betz OB, Betz VM, Nazarian A, et al. 2007. Delayed administration of adenoviral BMP2 vector improves the formation of bone in osseous defects. Gene Ther 14:1039-1044.

17. Kol A, Arzi B, Athanasiou KA, et al. 2015. Companion animals: Translational scientist's new best friends. Sci Trans1 Med 7:308ps321.

18. Cheng I, Goodman SB, Leucht P. 2014. If not BMP, then what? AAOS Now

http://www.aaos.org/news/aaosnow/dec14/research4.asp.

\section{Acknowledgements}

MJS is supported by the AO Foundation and European Community Seventh Framework Programme Grant, BioDesign (262948). CHE acknowledges support from the AO Foundation, US Department of Defense (W81XWH-10-1-0888 and W81XWH-13-0324), and NIH (NIAMS

RO1 AR050243) 